\title{
Accurate and Interpretable Regression Trees using Oracle Coaching
}

\author{
Ulf Johansson, Cecilia Sönströd, Rikard König \\ School of Business and IT \\ University of Borås, Sweden \\ Email: \{ulf.johansson, cecilia.sonstrod, rikard.konig\}@hb.se
}

\begin{abstract}
In many real-world scenarios, predictive models need to be interpretable, thus ruling out many machine learning techniques known to produce very accurate models, e.g., neural networks, support vector machines and all ensemble schemes. Most often, tree models or rule sets are used instead, typically resulting in significantly lower predictive performance. The overall purpose of oracle coaching is to reduce this accuracy vs. comprehensibility trade-off by producing interpretable models optimized for the specific production set at hand. The method requires production set inputs to be present when generating the predictive model, a demand fulfilled in most, but not all, predictive modeling scenarios. In oracle coaching, a highly accurate, but opaque, model is first induced from the training data. This model ("the oracle") is then used to label both the training instances and the production instances. Finally, interpretable models are trained using different combinations of the resulting data sets. In this paper, the oracle coaching produces regression trees, using neural networks and random forests as oracles. The experiments, using 32 publicly available data sets, show that the oracle coaching leads to significantly improved predictive performance, compared to standard induction. In addition, it is also shown that a highly accurate opaque model can be successfully used as a preprocessing step to reduce the noise typically present in data, even in situations where production inputs are not available. In fact, just augmenting or replacing training data with another copy of the training set, but with the predictions from the opaque model as targets, produced significantly more accurate and/or more compact regression trees.
\end{abstract}

Keywords-Oracle coaching, Regression trees, Predictive modeling, Interpretable models

\section{INTRODUCTION.}

The purpose of predictive modeling is to obtain a model that produces accurate predictions regarding some phenomenon, typically described in terms of attributes and a target concept. When viewed as a data mining problem, this entails finding and modeling patterns between an input, in the form of an attribute vector, and an output, in the form of a single target variable. When the target variable is real-valued, the task is a regression problem, while target values from a set of predefined labels result in a classification task. Both problem types have been extensively studied in machine learning and a wealth of techniques exist that produce models with good predictive performance. Generally, the best predictive

This work was supported by the Swedish Foundation for Strategic Research through the project High-Performance Data Mining for Drug Effect Detection (IIS11-0053), the Swedish Retail and Wholesale Development Council through the project Innovative Business Intelligence Tools (2013:5) and the Knowledge Foundation through the project Big Data Analytics by Online Ensemble Learning (20120192). performance is obtained using techniques that produce opaque models, such as artificial neural networks (ANNs), support vector machines (SVMs) or ensemble techniques, where it is not possible for a human to inspect the relationships found by the model. When predictive accuracy is the only property of interest, this is perfectly acceptable, but in many applications interpretable models are needed, making it necessary for techniques to produce transparent, rather than opaque, models. In a transparent model, relationships between input and output are made explicit in some easily interpretable representation and hence allow insights into the underlying domain to be gained. Generally, this possibility comes at the price of reduced predictive performance, compared to an opaque model. The situation where predictive performance is sacrificed in order to obtain an interpretable model is known as the accuracy vs. comprehensibility trade-off and has been discussed extensively in data mining research, see e.g., [1, 2]. Apart from the obvious situation where a model needs to be interpretable in order for its predictions to be verified for legal or safety reasons [3], it has been argued that interpretable models increase user acceptance, see e.g., [4, 5]. It is important to note that a transparent model is not necessarily interpretable viewed as an entity, due to it possibly containing hundreds or thousands of symbols. However, in a transparent model it is, in principle, always possible to follow the logic behind each and every prediction made.

Experimental evaluation of machine learning techniques always includes reporting performance on data not used to train the model. Usually, a fixed amount of data with known target values is available, and has to be used both to train and evaluate the model. In this situation, all results regarding predictive performance carry the assumption that the data used to evaluate the model has not been used at all during model building and parameter tuning. However, it is important to realize that this is a situation that is not typical for real-world applications. Instead, the usual state of affairs is that historical data with known target values are available to build a data mining model that will be applied to some production data with unknown target values, in order to solve a business problem. In these cases, the only thing that really matters is maximizing predictive accuracy on this production data. A key insight here is that often a large number of production instances will be available, and the predictive model is actually built especially for these instances. Examples of applications where this might occur are when selecting recipients of a mass marketing campaign from a customer register or deciding which are the most promising compounds from a newly synthesized molecule library to further investigate during drug development. It should also be 
noted that in many such instances, an interpretable model is desirable, or even mandatory.

We have previously suggested an approach called oracle coaching, especially aimed at increasing predictive performance on production data for transparent models. The idea is to build the transparent model in three steps:

1) build a high-performance opaque model using normal training data, i.e., the available labeled data

2) employ the opaque model to obtain predicted target values for both training and production instances

3) utilize the predicted values as training data for the transparent model, possibly in conjunction with the original training data

The term oracle coaching comes from the underlying idea of viewing the stronger opaque model as an oracle, whose predicted values are regarded as true values by the transparent model during training. So far, studies using oracle coaching have only been performed on classification problems, but since the setup only relies on the availability of high-performance opaque models to serve as oracles and ditto transparent models to utilize the oracle data, it is natural to extend the concept to regression problems. The main contribution of this paper is thus the novel application of the oracle coaching for regression tasks, using readily available state-of-the-art techniques, in order to obtain increased predictive performance for interpretable models.

\section{BACKGROUND.}

The most commonly used representation for transparent models is decision trees, and particularly for classification, these are almost ubiquitously used when interpretability is needed. However, as pointed out by Dobra and Gehrke in [6], even though regression is an important task in data mining, regression trees have not been extensively studied, with the notable exception of Breiman's CART [7] algorithm. They also note an increased interest in accurate and interpretable models for regression tasks. Despite this, more than a decade later, very few machine learning techniques aimed at producing interpretable regression models exist; with rule learning for regression, in particular, being an under-developed field [8].

Other than statistical methods, like (multiple) linear regression, the most popular data mining techniques used to produce high-performance models for regression problems are ANNs and SVMs. Both these types of models have repeatedly been shown to obtain robustly good predictive performance in a wide range of applications, despite both being rather sensitive to parameter settings. Naturally, given any technique producing regression models, it is also possible to build an ensemble predictor, using e.g., bagging [9], in order to further boost performance.

The insight that opaque models generally have superior predictive performance, but that interpretable models often are essential, has prompted research into rule extraction, aimed at extracting transparent models (typically trees or rule sets) from opaque models. In [10], Craven and Shavlik propose five criteria for evaluating rule extraction algorithms: comprehensibility (i.e., interpretability), accuracy, fidelity, scalability and generality. Fidelity is the key property that the extracted model faithfully mimics the opaque model's predictions. Originally, research in rule extraction focused exclusively on ANNs as opaque models, and many early rule extraction algorithms explicitly utilized the underlying ANN architecture, building transparent models based on the trained network's nodes and weights. Since this requires "seeing into" the network, this strategy is called open- or white-box rule extraction. When employing open-box rule extraction, the primary concern is to (in detail) explain how the opaque neural network model produces its predictions. One main drawback of open-box rule extraction is the inherent reliance on the type of opaque model used, making general applicability very limited. Furthermore, the resulting transparent models tend to be quite complex, since fidelity is prioritized over interpretability. A contrasting strategy is to accept that the opaque model is indeed opaque and perform black-box rule extraction, where the aim is to reproduce the behaviour of the opaque model in terms only of capturing the connection between input and output. The main advantage of using black-box rule extraction algorithms is, of course, that they can be applied to all kinds of opaque models.

An interesting discussion about the purpose of rule extraction is found in [11], where Zhou describes rule extraction as two very different tasks; rule extraction for neural networks and rule extraction using neural networks. While the first task is solely aimed at understanding the inner workings of an opaque model, the second task is explicitly aimed at extracting a comprehensible model with higher accuracy than a comprehensible model created directly from the data set. More specifically, in rule extraction for opaque models, the purpose is most often to explain the reasoning behind individual predictions from an opaque model, i.e., the actual predictions are still made by the opaque model. In rule extraction using opaque models, the predictions are made by the extracted model, so it is used both as the predictive model and as a tool for understanding and analysis of the underlying relationship. In that situation, predictive performance is what matters, so the data miner must have reasons to believe that the extracted model will be more accurate than other comprehensible models induced directly from the data. The motivation for that rule extraction using opaque models may work is that even a very complex and highly accurate opaque model is a smoothed representation of the underlying relationship. In fact, training instances misclassified by the opaque model are often atypical, i.e., learning such instances will reduce the generalization capability. Indeed it is fair to say that, in many cases, a good opaque model is a better representation of a data set than the actual data instances themselves.

\section{A. Related work.}

The concept of oracle coaching was introduced in a rule extraction context in [12], with the explicit aim of increasing predictive performance for the extracted model. Since then, a number of studies have been published, thoroughly evaluating [13-15] and further developing the technique [16]. In these studies, ensembles using bagging [9] of both multiple-layer perceptrons (MLPs) and radial basis function (RBF) networks, as well as random forest trees [17] have been used as oracle models. Transparent model representations have been decision trees and decision lists. Extensive evaluation in these studies, both using benchmark data sets and a large number of data sets from the medicinal chemistry domain, have demonstrated 
that utilizing production instances labeled by an oracle opaque model increases predictive performance for the transparent model. In particular, augmenting normal training data with labeled production instances will all but guarantee a significant increase in predictive accuracy for the transparent model. Using only labeled production data as training instances for the transparent model does often result in smaller models, but typically with a lower gain in accuracy. As can be expected, increasing the amount of training data with both label training instances and labeled production instances will result in increased model size, but most often without a significant gain in accuracy. Another notable result is that further gains in transparent model accuracy can be obtained by optimizing the predictive performance of the oracle, rather than fidelity. Together, the previous work in this area shows that oracle coaching for classification is both versatile and robust.

\section{METHOD.}

As mentioned in the introduction, the purpose of this study is to evaluate the use of highly accurate opaque models serving as coaching oracle when creating transparent regression models. More specifically, regression trees induced directly from training data are compared to regression trees built using different combinations of training data and production data. Naturally, the hypothesis is that the models produced using oracle coaching will be more accurate, more compact or both.

In this study, two kinds of predictive models are used as oracles, random forests [17] and multi-layer perceptron neural networks. CART regression trees [7], as implemented in the MatLab statistics toolbox are used as transparent models. All parameters were left at their default values, with the exception of QEToler, which was set to 0.001, in order to produce slightly more compact CART trees. Identical settings were used over all data sets and, when applicable, methods. Hence, all random forests consisted of 500 random trees. Similarly, all ANNs had one hidden layer with exactly 20 units. All experimentation was performed in MatLab, using the Neural network and the Statistics toolboxes.

Naturally, the opaque models (the random forest or the ANN) are first generated using training data only. This model (the oracle) is then applied to both the training instances and the production instances, thus creating predictions for both training and production data. This results in three different data sets:

- The training data, consisting of the original training data set, i.e., original input vectors with corresponding correct target values.

- The extraction data, consisting of the instances in the original training data set, i.e., original input vectors, where target values have been replaced with predictions from the opaque model.

- The oracle data, consisting of the production instances with predictions from the opaque model as target values.

In the experimentation, we evaluate using all different combinations of these data sets when generating the final regression tree:
- Induction (I): Standard tree induction using original training data only. Should maximize training accuracy.

- Extraction (E): Standard tree extraction; i.e., using extraction data only. Should maximize training fidelity.

- Explanation (X): Uses only oracle data, i.e., should maximize production fidelity.

- Exduction ${ }^{1}$ (IE): Uses training data and extraction data, so this data set will contain two instances with identical input vectors but different targets; one is the true target and one is the prediction from the opaque model. It should be noted that this setup does not use oracle data, thus not requiring the production input vectors when building the model. This setup should maximize a combination of training accuracy and fidelity.

- Indanation (IX): Uses training data and oracle data, i.e., maximizes training accuracy and production fidelity.

- Extanation (EX): Uses extraction data and oracle data, i.e., maximizes fidelity towards the opaque model on both training and production data.

- Indextanation (IEX): Uses all three data sets; i.e., will maximize training accuracy, training fidelity and test fidelity simultaneously.

Before the experimentation, all target values were normalized to $[0,1]$, in order to obtain more readable error measure comparisons across data sets. In this study, both the root-meansquare-error (RMSE) and the Pearson correlation coefficient (r) are used to measure model accuracy. Model sizes are measured using the total number of nodes. For the actual evaluation, 10x10-fold cross-validation was employed, i.e., all results reported are average values over the 100 folds.

The 32 publicly available data sets used in the experimentation are small to medium sized; ranging from approximately 500 to 10000 instances. All but one data set are from the UCI [18], Delve [19] or KEEL [20] repositories. The data sets are described in Table I below, where \#inst. is the number of instances and \#attrib. is the number of input attributes.

\section{TABLE I. DATA SETS}

\begin{tabular}{lccc|lccc} 
Name & \#inst. & \#attrib. & Origin & Name & \#inst. & \#attrib. & Origin \\
\hline abalone & 4177 & 8 & UCI & kin8fm & 8192 & 8 & Delve \\
anacalt & 566 & 7 & KEEL & kin8nh & 8192 & 8 & Delve \\
bank8fh & 8192 & 8 & Delve & kin8nm & 8192 & 8 & Delve \\
bank8fm & 8192 & 8 & Delve & laser & 993 & 4 & KEEL \\
bank8nh & 8192 & 8 & Delve & mg & 1385 & 6 & {$[21]$} \\
bank8nm & 8192 & 8 & Delve & mortage & 1048 & 15 & KEEL \\
boston & 506 & 13 & UCI & plastic & 1055 & 2 & KEEL \\
comp & 8192 & 12 & Delve & puma8fh & 8192 & 8 & Delve \\
concreate & 992 & 8 & UCI & puma8fm & 8192 & 8 & Delve \\
cooling & 768 & 8 & UCI & puma8nh & 8192 & 8 & Delve \\
deltaA & 7129 & 5 & KEEL & puma8nm & 8192 & 8 & Delve \\
deltaE & 9517 & 6 & KEEL & quakes & 2178 & 2 & KEEL \\
friedm & 1200 & 5 & KEEL & treasury & 1048 & 15 & KEEL \\
heating & 768 & 8 & UCI & wineRed & 1359 & 11 & UCI \\
istanbul & 536 & 7 & UCI & wineWhite & 3961 & 11 & UCI \\
kin8fh & 8192 & 8 & Delve & wizmir & 1460 & 2 & KEEL
\end{tabular}

\footnotetext{
${ }^{1}$ The following names, combining the terms induction, extraction and explanation, are of course made up.
} 


\section{RESUlTS.}

Starting with Experiment 1, using ANN oracles, Table II below shows the results for the three different setups not utilizing oracle data. For comparison, the predictive performance of the ANNs extracted from are included in the table in the column named $\mathrm{O}$. Looking at the accuracy results, we see that IE is the most successful setup. This is of course a very interesting observation, showing that in scenarios where interpretable models are necessary, more accurate transparent models can be obtained by augmenting the training data with extracted data. Comparing I and E, there are very small differences in accuracy between inducing and extracting models.

When looking at models sizes, however, we see that the extracted models are by far the smallest. This is a clear indication that the general rule extraction paradigm using opaque models is able to reduce the noise typically present in data.

TABLE II. EXPERIMENT 1: ACCURACY RESULTS AND SIZE FOR INDUCTION AND EXTRACTION FROM ANN

\begin{tabular}{|c|c|c|c|c|c|c|c|c|c|c|c|}
\hline & \multicolumn{4}{|c|}{ RMSE } & \multicolumn{4}{|c|}{ Correlation (r) } & \multicolumn{3}{|c|}{ Size } \\
\hline & I & $\mathrm{E}$ & IE & $\mathrm{O}$ & I & $\mathrm{E}$ & IE & $\mathrm{O}$ & I & E & IE \\
\hline abalone & .095 & .079 & .082 & .077 & .628 & .725 & .705 & .742 & 765 & 213 & 772 \\
\hline anacalt & .069 & .067 & .065 & .076 & 946 & .947 & .951 & .936 & 50 & 42 & 74 \\
\hline bank8fh & .106 & .094 & .094 & .090 & .816 & .854 & .854 & .867 & 807 & 117 & 521 \\
\hline bank8fm & .049 & .054 & .050 & .041 & 965 & .959 & .964 & .976 & 186 & 116 & 157 \\
\hline bank8nh & .138 & .114 & .119 & .109 & .518 & .638 & .608 & .674 & 1160 & 306 & 1095 \\
\hline bank8nm & .065 & .067 & .064 & .049 & .864 & .855 & .868 & .925 & 424 & 319 & 421 \\
\hline boston & .094 & .097 & .095 & .082 & .887 & .876 & .884 & .913 & 134 & 116 & 158 \\
\hline comp & .037 & .042 & .038 & .038 & .980 & .974 & .979 & .978 & 125 & 69 & 100 \\
\hline concreate & .082 & .101 & .085 & .088 & .916 & .866 & .908 & .900 & 229 & 172 & 281 \\
\hline cooling & .056 & .073 & .059 & .073 & .976 & .958 & .974 & .959 & 90 & 48 & 113 \\
\hline deltaA & .044 & .042 & .040 & .040 & .789 & .804 & .823 & .823 & 774 & 190 & 631 \\
\hline deltaE & .061 & .055 & .055 & .054 & .740 & .782 & .785 & .789 & 1181 & 171 & 884 \\
\hline friedm & .098 & .095 & .094 & .051 & .857 & .861 & .865 & .962 & 307 & 259 & 313 \\
\hline heating & .025 & .056 & .035 & .058 & .996 & .979 & .992 & .976 & 45 & 41 & 61 \\
\hline istanbul & .104 & .081 & .088 & .082 & .565 & .704 & .656 & .707 & 206 & 103 & 311 \\
\hline kin8fh & .095 & .091 & .091 & .070 & .735 & .752 & .755 & .859 & 877 & 497 & 760 \\
\hline kin8fm & .075 & .076 & .075 & .031 & .860 & .857 & .862 & .977 & 531 & 500 & 527 \\
\hline kin8nh & .157 & .144 & .144 & 2.53 & 1.03 & 2.44 & .609 & .737 & 1271 & 706 & 1247 \\
\hline kin8nm & .139 & 138 & .135 & .091 & .692 & .680 & .698 & .872 & 973 & 798 & 993 \\
\hline laser & .052 & .057 & .051 & .037 & .956 & .949 & .958 & .977 & 106 & 99 & 116 \\
\hline $\mathrm{mg}$ & .097 & .117 & .094 & .109 & .924 & .886 & .926 & .901 & 176 & 109 & 237 \\
\hline mortage & .024 & .026 & .024 & .011 & .994 & .993 & .994 & .999 & 39 & 40 & 41 \\
\hline plastic & .179 & .162 & .158 & .145 & .861 & .885 & .891 & .909 & 339 & 129 & 282 \\
\hline puma8fh & .172 & 143 & .148 & .141 & 682 & .777 & .759 & .783 & 1116 & 92 & 855 \\
\hline puma8fm & .068 & .073 & .069 & .063 & .968 & .963 & .967 & .972 & 178 & 82 & 125 \\
\hline puma8nh & .156 & 138 & .137 & .137 & .755 & .803 & .805 & .806 & 930 & 148 & 728 \\
\hline puma8nm & .063 & .076 & .066 & .066 & .969 & .956 & .967 & .967 & 183 & 132 & 170 \\
\hline stock & .041 & .049 & .042 & .039 & .984 & .978 & .983 & .986 & 82 & 71 & 82 \\
\hline treasury & .023 & .023 & .022 & .015 & .992 & .993 & .993 & .997 & 35 & 33 & 35 \\
\hline wineRed & .168 & 133 & .143 & .133 & .440 & .587 & .534 & .593 & 456 & 224 & 672 \\
\hline wineWhite & .151 & 126 & .129 & .123 & .435 & .533 & .521 & .562 & 1036 & 309 & 1227 \\
\hline wizmir & .034 & .035 & .035 & .024 & .990 & .989 & .989 & .994 & 60 & 52 & 56 \\
\hline Mean & .088 & .085 & .082 & .074 & .820 & .843 & .845 & .876 & 465 & 197 & 439 \\
\hline Mean rank & 2.19 & 2.22 & 1.59 & & 2.16 & 2.31 & 1.53 & & 2.53 & 1.03 & 2.44 \\
\hline
\end{tabular}

In order to further analyze these results, and to find out if there are any statistically significant differences, we used the recommended procedure in [22] and performed a Friedman test [23], followed by Bergmann-Hommel's [24] dynamic procedure to establish all pairwise differences. Table III shows the resulting adjusted p-values. Significant results for $\alpha=0.05$ are given in bold. As expected, most of the differences are indeed significant, at this level. Specifically, IE obtained a significantly lower RMSE and a significantly higher correlation coefficient than both I and E. E, on the other hand, produced significantly smaller models than both I and IE.

TABLE III. EXPERIMENT 1: INDUCTION AND EXTRACTION FROM ANN. AdJusted P-VALUES USING BERGMANN'S PROCEDURE

\begin{tabular}{lccc} 
& RMSE & r & Size \\
\hline IE vs. E & $\mathbf{0 . 0 4 4}$ & $\mathbf{0 . 0 1 5}$ & $\mathbf{1 . 9 E - 0 8}$ \\
IE vs. I & $\mathbf{0 . 0 4 4}$ & $\mathbf{0 . 0 1 5}$ & 0.708 \\
E vs. I & 0.851 & 0.708 & $\mathbf{5 . 9 E - 0 9}$
\end{tabular}

Turning to the second part of Experiment 1, i.e., when the opaque models are random forests, Table IV below shows the accuracies and model sizes. First, it should be noted that the random forests are generally more accurate than the ANNs in the previous part, but the differences are often quite small. Nevertheless, the rule extraction clearly benefits from the slightly more accurate random forests. Specifically comparing the evaluated setups, standard rule extraction (E) is actually the most accurate setup on a large number of data sets. When considering all three setups over all data sets, however, IE again obtained the lowest mean rank for both accuracy metrics.

Regarding tree sizes, E again produced much smaller models than the other two setups, confirming the reasoning about the ability to filter out anomalies in the training data.

TABLE IV. EXPERIMENT 1: ACCURACY RESULTS AND SIZE FOR INDUCTION AND EXTRACTION FROM RF

\begin{tabular}{|c|c|c|c|c|c|c|c|c|c|c|c|}
\hline & \multicolumn{4}{|c|}{ RMSE } & \multicolumn{4}{|c|}{ Correlation (r) } & \multicolumn{3}{|c|}{ Size } \\
\hline & I & $\mathrm{E}$ & IE & $\mathrm{O}$ & I & E & IE & $\mathrm{O}$ & $\mathrm{I}$ & $\mathrm{E}$ & IE \\
\hline abalone & .095 & .079 & .086 & .076 & .628 & .725 & .681 & .747 & 765 & 299 & 689 \\
\hline & .069 & .068 & .068 & .074 & .946 & .956 & .948 & .949 & 50 & 25 & 65 \\
\hline & .106 & .093 & .096 & .092 & .816 & .856 & .845 & .862 & 807 & 193 & 562 \\
\hline & .049 & .053 & .050 & .043 & .965 & .960 & .964 & .976 & 186 & 111 & 151 \\
\hline & .138 & .114 & .123 & .110 & .518 & .642 & .581 & .670 & 1160 & 487 & 1035 \\
\hline & .065 & .064 & .063 & .051 & .864 & .867 & .871 & .926 & 424 & 260 & 368 \\
\hline 2 & .094 & .093 & .091 & .079 & .887 & .886 & .892 & .921 & 134 & 87 & 130 \\
\hline$m p$ & .037 & .039 & .037 & .029 & .980 & .978 & .979 & .988 & 125 & 63 & 94 \\
\hline reate & .082 & .086 & .079 & .073 & .916 & .906 & .919 & .942 & 229 & 158 & 222 \\
\hline & .056 & .055 & .052 & .049 & .976 & .977 & .980 & .982 & 90 & 35 & 72 \\
\hline$A$ & .044 & .039 & .040 & .037 & .789 & .831 & .821 & .848 & 774 & 211 & 566 \\
\hline & .061 & .054 & .055 & .053 & .740 & .792 & .778 & .800 & 1181 & 210 & 850 \\
\hline & .098 & .094 & .093 & .069 & .857 & .864 & .867 & .938 & 307 & 206 & 287 \\
\hline & .025 & .035 & .026 & .026 & .996 & .991 & .995 & .996 & 45 & 41 & 46 \\
\hline & .104 & .082 & .093 & .079 & .565 & 697 & .618 & .722 & 206 & 140 & 275 \\
\hline & .095 & .090 & .091 & .075 & .735 & .756 & .752 & .848 & 877 & 486 & 732 \\
\hline & .075 & .075 & .075 & .046 & .860 & .859 & .861 & .963 & 531 & 404 & 484 \\
\hline & .157 & .140 & .145 & .126 & .569 & .630 & 607 & .720 & 1271 & 677 & 1124 \\
\hline & .139 & .130 & .131 & .105 & .692 & .716 & .714 & .844 & 973 & 615 & 874 \\
\hline & .052 & .050 & .050 & .036 & .956 & .960 & .960 & .979 & 106 & 91 & 107 \\
\hline & 097 & .097 & .092 & .089 & .924 & .923 & .930 & .936 & 176 & 127 & 170 \\
\hline ort & .024 & .026 & .025 & .012 & .994 & .993 & .994 & .999 & 39 & 38 & 39 \\
\hline & .179 & .174 & .165 & .161 & .861 & .872 & .880 & .896 & 339 & 119 & 312 \\
\hline & .172 & .144 & .155 & .143 & .682 & .774 & .734 & .775 & 1116 & 301 & 866 \\
\hline & .068 & .071 & .069 & .063 & .968 & .964 & .967 & .972 & 178 & 89 & 128 \\
\hline & .156 & .134 & .141 & .133 & .755 & .816 & .793 & .819 & 930 & 252 & 703 \\
\hline & .063 & .069 & .065 & .059 & .969 & .964 & .968 & .976 & 183 & 120 & 156 \\
\hline & .041 & .044 & .041 & .033 & .984 & .982 & .984 & .991 & 82 & 64 & 73 \\
\hline & .023 & .023 & .023 & .013 & .992 & .993 & .993 & 997 & 35 & 31 & 33 \\
\hline & .168 & .136 & .151 & .128 & .440 & .570 & .497 & .628 & 456 & 315 & 557 \\
\hline wineWhite & .151 & .124 & .135 & .116 & .435 & .553 & .490 & .621 & 1036 & 588 & 1007 \\
\hline wizmir & .034 & .037 & .035 & .022 & .990 & .988 & .989 & .996 & 60 & 47 & 55 \\
\hline & .088 & .082 & .083 & .072 & .820 & .851 & .839 & .882 & 465 & 215 & 401 \\
\hline 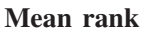 & 2.47 & 1.88 & 1.66 & 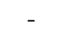 & 2.41 & 1.91 & 1.69 & 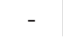 & 2.84 & 1.00 & 2.16 \\
\hline
\end{tabular}

Table V below shows adjusted p-values from Bergmann's procedure. Significant results (for $\alpha=0.05$ ) are again given in 
bold. Comparing these results (obtained using random forests) to the results produced using ANNs, the most important difference is that using standard rule extraction $(\mathrm{E})$ is now a very strong choice. $\mathrm{E}$ is actually significantly more accurate than standard induction (I), and the resulting models are most often as accurate as the ones produced using IE. This is despite the fact that the regression trees built by $\mathrm{E}$ are significantly smaller than the other two setups.

TABLE V. EXPERIMENT 1: INDUCTION AND EXTRACTION FROM RF. ADJUSTED P-VALUES USING BERGMANN'S PROCEDURE

\begin{tabular}{lccc} 
& RMSE & r & Size \\
\hline IE vs. E & 0.574 & 0.382 & $\mathbf{3 . 7 E - 0 6}$ \\
IE vs. I & $\mathbf{0 . 0 1 5}$ & $\mathbf{0 . 0 1 2}$ & $\mathbf{0 . 0 0 6}$ \\
E vs. I & $\mathbf{0 . 0 2 4}$ & $\mathbf{0 . 0 4 6}$ & $\mathbf{4 . 9 E - 1 3}$
\end{tabular}

Summarizing Experiment 1, the most important finding is that the use of a highly accurate opaque model as a preprocessing step is clearly beneficial for generating transparent regression models. The most accurate setup overall combined original training data, with training data labeled by the opaque model, in the generation phase. In addition, utilizing data labeled by the opaque model also produced significantly smaller regression trees.

Turning to Experiment 2, i.e., when the oracle data are included, Table VIII, placed after the references section, shows accuracy results and tree sizes using ANNs as oracles. The most obvious result is the benefit from utilizing oracle data. More specifically, setups combining oracle data with training data (IX and IEX) are the most accurate. Interestingly enough, these setups are more accurate than X, i.e., using only the oracle data. $\mathrm{X}$ is actually even slightly less accurate than EX, which combines extracted data with oracle data. Still, the three setups not using oracle data (I, E, IE) have the worst accuracy, so the oracle coaching procedure paid off. Looking at tree sizes, $\mathrm{X}$ produced the most compact trees for all data sets. With this in mind, if the purpose is to explain or analyse the predictions for a specific production set, setup $\mathrm{X}$ is a viable choice. Another important observation is that setups using extracted data $(\mathrm{E}, \mathrm{EX})$ produce much smaller models than setups generated on training data. This confirms the findings in Experiment 1 that highly accurate opaque models can serve as pre-processing tools when building transparent predictive models. Finally, it should be noted that the increased accuracy of IEX and IX do not result in more complex trees, compared to I.

Table VI below, which shows adjusted p-values from Bergmann's procedure, confirms most of the observed differences to be statistically significant. Specifically, both setups combining training and oracle data (IX and IEX) are significantly more accurate than all three setups not utilizing oracle data (I, E, IE). Regarding model sizes, the setups using only oracle data $(\mathrm{E}, \mathrm{EX}$ and $\mathrm{X})$ all produced significantly smaller trees than setups including original training data, i.e., I, IX and IEX.
TABLE VI. EXPERIMENT 2: ANNS AS ORACLES. ADJUSTED P-VALUES USING BERGMANN'S PROCEDURE

\begin{tabular}{lccc} 
& RMSE & $\mathrm{r}$ & Size \\
\hline IX vs. E & $\mathbf{7 . 4 E - 0 6}$ & $\mathbf{9 . 6 E - 0 7}$ & $\mathbf{1 . 9 E - 1 0}$ \\
IEX vs. E & $\mathbf{9 . 7 E - 0 6}$ & $\mathbf{2 . 5 E - 0 6}$ & $\mathbf{1 . 9 E - 0 7}$ \\
IX vs. I & $\mathbf{6 . 3 E - 0 5}$ & $\mathbf{4 . 8 E - 0 5}$ & 1.346 \\
IEX vs. I & $\mathbf{7 . 3 E - 0 5}$ & $\mathbf{9 . 6 E - 0 5}$ & 1.930 \\
EX vs. E & $\mathbf{0 . 0 0 3}$ & $\mathbf{0 . 0 0 3}$ & 1.154 \\
IX vs. IE & $\mathbf{0 . 0 1 8}$ & $\mathbf{0 . 0 1 5}$ & 1.346 \\
EX vs. I & $\mathbf{0 . 0 1 8}$ & $\mathbf{0 . 0 3 5}$ & $\mathbf{2 . 6 E - 0 5}$ \\
X vs. E & $\mathbf{0 . 0 1 8}$ & $\mathbf{0 . 0 1 0}$ & 0.195 \\
IEX vs. IE & $\mathbf{0 . 0 1 8}$ & $\mathbf{0 . 0 2 0}$ & 1.930 \\
X vs. I & 0.051 & 0.084 & $\mathbf{3 . 1 E - 1 5}$ \\
IX vs. X & 0.442 & 0.216 & $\mathbf{6 . 3 E - 1 8}$ \\
IE vs. E & 0.442 & 0.216 & $\mathbf{1 . 8 E - 0 8}$ \\
IEX vs. X & 0.442 & 0.216 & $\mathbf{9 . 8 E - 1 4}$ \\
EX vs. IE & 0.467 & 0.869 & $\mathbf{2 . 6 E - 0 5}$ \\
EX vs. IX & 0.700 & 0.320 & $\mathbf{8 . 1 E - 0 7}$ \\
IE vs. I & 0.700 & 0.444 & 1.930 \\
IEX vs. EX & 0.700 & 0.320 & $\mathbf{1 . 5 E - 0 4}$ \\
X vs. IE & 0.707 & 0.934 & $\mathbf{3 . 7 E - 1 5}$ \\
E vs. I & 1.868 & 1.254 & $\mathbf{1 . 8 E - 0 8}$ \\
EX vs. X & 1.868 & 1.457 & $\mathbf{0 . 0 0 3}$ \\
IEX vs. IX & 1.868 & 1.457 & 1.346
\end{tabular}

The results for Experiment 2 when using the random forest as the oracle, are presented in Table IX, at the end of the paper. Again, we see that setups using oracle data are the most accurate. The most interesting observation is perhaps that EX here performs relatively better than when using ANNs. The reason is, of course, the slightly stronger oracle. Actually, in this setting, EX is almost as accurate as IX, despite the fact that the trees are (on average) less than half the size.

As seen in Table VII below, there are a large number of statistically significant differences for $\alpha=0.05$. Specifically, all three setups combining training and/or extraction data with oracle data (IX, EX, IEX) are significantly more accurate than all setups not utilizing oracle data, i.e., I, E and IE.

TABLE VII. EXPERIMENT 2: RFS AS ORACLES. AdJUSTED P-VALUES USING BERGMANN'S PROCEDURE

\begin{tabular}{lccc} 
& RMSE & r & Size \\
\hline IX vs. E & $\mathbf{8 . 7 E - 0 4}$ & $\mathbf{0 . 0 0 2}$ & $\mathbf{6 . 4 E - 1 0}$ \\
IEX vs. E & $\mathbf{0 . 0 0 4}$ & $\mathbf{0 . 0 0 3}$ & $\mathbf{4 . 2 E - 0 4}$ \\
IX vs. I & $\mathbf{4 . 9 E - 0 7}$ & $\mathbf{4 . 6 E - 0 6}$ & 2.317 \\
IEX vs. I & $\mathbf{5 . 3 E - 0 6}$ & $\mathbf{1 . 3 E - 0 5}$ & $\mathbf{0 . 0 4 6}$ \\
EX vs. E & $\mathbf{0 . 0 0 4}$ & $\mathbf{0 . 0 0 2}$ & 2.317 \\
IX vs. IE & $\mathbf{8 . 7 E - 0 4}$ & $\mathbf{0 . 0 0 2}$ & 0.060 \\
EX vs. I & $\mathbf{5 . 3 E - 0 6}$ & $\mathbf{4 . 0 E - 0 6}$ & $\mathbf{1 . 9 E - 1 0}$ \\
X vs. E & 0.091 & $\mathbf{0 . 0 2 1}$ & $\mathbf{0 . 0 2 9}$ \\
IEX vs. IE & $\mathbf{0 . 0 0 4}$ & $\mathbf{0 . 0 0 4}$ & 2.317 \\
X vs. I & $\mathbf{8 . 7 E - 0 4}$ & $\mathbf{2 . 3 E - 0 4}$ & $\mathbf{1 . 6 E - 2 0}$ \\
IX vs. X & 1.004 & 2.378 & $\mathbf{6 . 0 E - 2 0}$ \\
IE vs. E & 2.410 & 2.914 & $\mathbf{1 . 2 E - 0 4}$ \\
IEX vs. X & 1.086 & 2.378 & $\mathbf{6 . 3 E - 1 1}$ \\
EX vs. IE & $\mathbf{0 . 0 0 4}$ & $\mathbf{0 . 0 0 2}$ & $\mathbf{1 . 0 E - 0 4}$ \\
EX vs. IX & 2.410 & 2.914 & $\mathbf{4 . 2 E - 1 0}$ \\
IE vs. I & 1.004 & 1.192 & 0.060 \\
IEX vs. EX & 2.410 & 2.914 & $\mathbf{3 . 1 E - 0 4}$ \\
X vs. IE & 0.091 & $\mathbf{0 . 0 3 0}$ & $\mathbf{7 . 9 E - 1 2}$ \\
E vs. I & 1.004 & 1.283 & $\mathbf{3 . 1 E - 1 0}$ \\
EX vs. X & 1.086 & 2.378 & $\mathbf{0 . 0 3 2}$ \\
IEX vs. IX & 2.410 & 2.914 & $\mathbf{0 . 0 4 6}$
\end{tabular}

Based on the correlation coefficient $\mathrm{r}, \mathrm{X}$ is also significantly more accurate than the three setups not using oracle 
data. Using RMSE, however, the superior accuracy is only significant against I. Looking at model sizes, the picture is almost identical to when ANNs are used as oracles, i.e., all setups using extracted data (but not training data) produced significantly smaller trees than setups using training data.

From these results, it is obvious that the oracle coaching paradigm works as intended in the regression context. Specifically, setups including oracle data are most often significantly more accurate than setups using only training or extraction data. Furthermore, generating models on the smoothed version of the training data (the extraction data), will lead to significantly more compact models, compared to using original training data. Interestingly enough, this study indicates that the quality of the oracle is not vital, as long as it is accurate enough. Specifically, the fairly large differences in accuracy between the ANNs and the random forests, did not carry over to the coached regression trees.

\section{CONCLUDING REMARKS.}

We have in this paper introduced oracle coaching for predictive regression. The purpose of this method is to produce interpretable models, and it applies to the very common situation where the predictive model is built for a specific production set with known input vectors. In oracle coaching, a highly accurate, but opaque, model ("the oracle") is first induced from standard training data. The oracle is then used to label both training instances and production instances. In the last step, interpretable models are trained using different combinations of these data sets. The method is very general, since any opaque model can serve as the oracle and any technique producing transparent models can benefit from this procedure. The experimental results show that for predictive regression, the use of oracle coaching produced significantly more accurate regression trees, compared to using standard induction. In addition, the results also show that even in situations where the production set inputs are not available, using an oracle as a pre-processing step, just to reduce the noise in the training data, produced significantly more accurate and/or smaller regression trees.

\section{REFERENCES}

[1] P. Domingos, "Knowledge discovery via multiple models," Intelligent Data Analysis, vol. 2, pp. 187-202, 1998.

[2] R. Blanco-Vega, J. Hernández-Orallo, and M. RamírezQuintana, "Analysing the trade-off between comprehensibility and accuracy in mimetic models," in Discovery Science, ser. LNCS. Springer, 2004, vol. 3245, pp. 338346.

[3] R. Andrews, J. Diederich, and A. B. Tickle, "Survey and critique of techniques for extracting rules from trained artificial neural networks," Knowl.-Based Syst., vol. 8, no. 6, pp. 373-389, 1995.

[4] M. W. Craven and J. W. Shavlik, "Extracting treestructured representations of trained networks," in $A d$ vances in Neural Information Processing Systems. MIT Press, 1996, pp. 24-30.

[5] A. A. Freitas, "A survey of evolutionary algorithms for data mining and knowledge discovery," in Advances in Evolutionary Computation. Springer-Verlag, 2002.
[6] A. Dobra and J. Gehrke, "Secret: A scalable linear regression tree algorithm," in KDD. New York, NY, USA: ACM, 2002, pp. 481-487.

[7] L. Breiman, J. Friedman, C. J. Stone, and R. A. Olshen, Classification and Regression Trees. Chapman \& Hall/CRC, 1984.

[8] J. Fürnkranz, D. Gamberger, and N. Lavrač, Foundations of Rule Learning. Springer Publishing Company, 2012.

[9] L. Breiman, "Bagging predictors," Machine Learning, vol. 24, no. 2, pp. 123-140, 1996.

[10] M. Craven and J. Shavlik, "Rule extraction: Where do we go from here?" University of Wisconsin Machine Learning Research Group working Paper 99-1, 1999.

[11] Z.-H. Zhou, "Rule extraction: using neural networks or for neural networks?" J. Comput. Sci. Technol., vol. 19, no. 2, pp. 249-253, 2004.

[12] U. Johansson, T. Löfström, R. König, and L. Niklasson, "Why not use an oracle when you got one?" Neural Information Processing: Letters and Reviews, vol. 10, no. 8-9, pp. 227-236, 2006.

[13] U. Johansson and L. Niklasson, "Evolving decision trees using oracle guides," in CIDM. IEEE, 2009, pp. 238244.

[14] U. Johansson, C. Sönströd, and T. Löfström, "Oracle coached decision trees and lists," in IDA, ser. LNCS, vol. 6065. Springer, 2010, pp. 67-78.

[15] C. Sönströd, U. Johansson, H. Boström, and U. Norinder, "Pin-pointing concept descriptions." in SMC. IEEE, 2010, pp. 2956-2963.

[16] U. Johansson, C. Sönströd, T. Löfström, and H. Boström, "Obtaining accurate and comprehensible classifiers using oracle coaching," Intell. Data Anal., vol. 16, no. 2, pp. 247-263, 2012.

[17] L. Breiman, "Random forests," Machine Learning, vol. 45, no. 1, pp. 5-32, 2001.

[18] K. Bache and M. Lichman, "UCI machine learning repository," 2013. [Online]. Available: http://archive.ics. uci.edu/ml

[19] C. E. Rasmussen, R. M. Neal, G. Hinton, D. van Camp, M. Revow, Z. Ghahramani, R. Kustra, and R. Tibshirani, "Delve data for evaluating learning in valid experiments," www. cs. toronto. edu/delve, 1996.

[20] J. Alcalá-Fdez, A. Fernández, J. Luengo, J. Derrac, and S. García, "Keel data-mining software tool: Data set repository, integration of algorithms and experimental analysis framework," Multiple-Valued Logic and Soft Computing, vol. 17, no. 2-3, pp. 255-287, 2011.

[21] G. W. Flake and S. Lawrence, "Efficient svm regression training with smo," Machine Learning, vol. 46, no. 1-3, pp. 271-290, 2002.

[22] S. García and F. Herrera, "An extension on statistical comparisons of classifiers over multiple data sets for all pairwise comparisons," Journal of Machine Learning Research, vol. 9, no. 2677-2694, p. 66, 2008.

[23] M. Friedman, "The use of ranks to avoid the assumption of normality implicit in the analysis of variance," Journal of American Statistical Association, vol. 32, pp. 675-701, 1937.

[24] B. Bergmann and G. Hommel, "Improvements of general multiple test procedures for redundant systems of hypotheses," in Multiple Hypotheses Testing. Springer, 1988, pp. 100-115. 


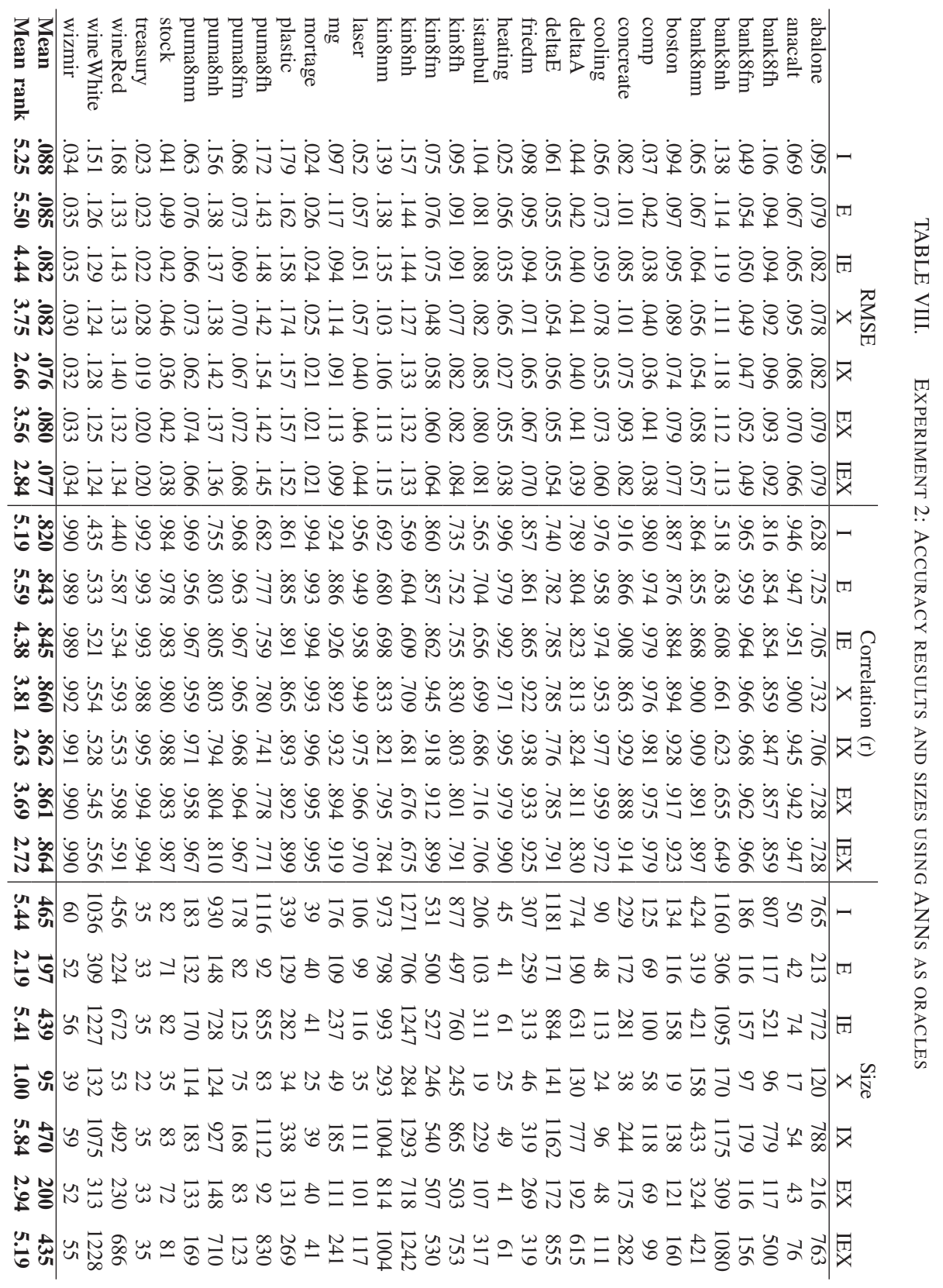




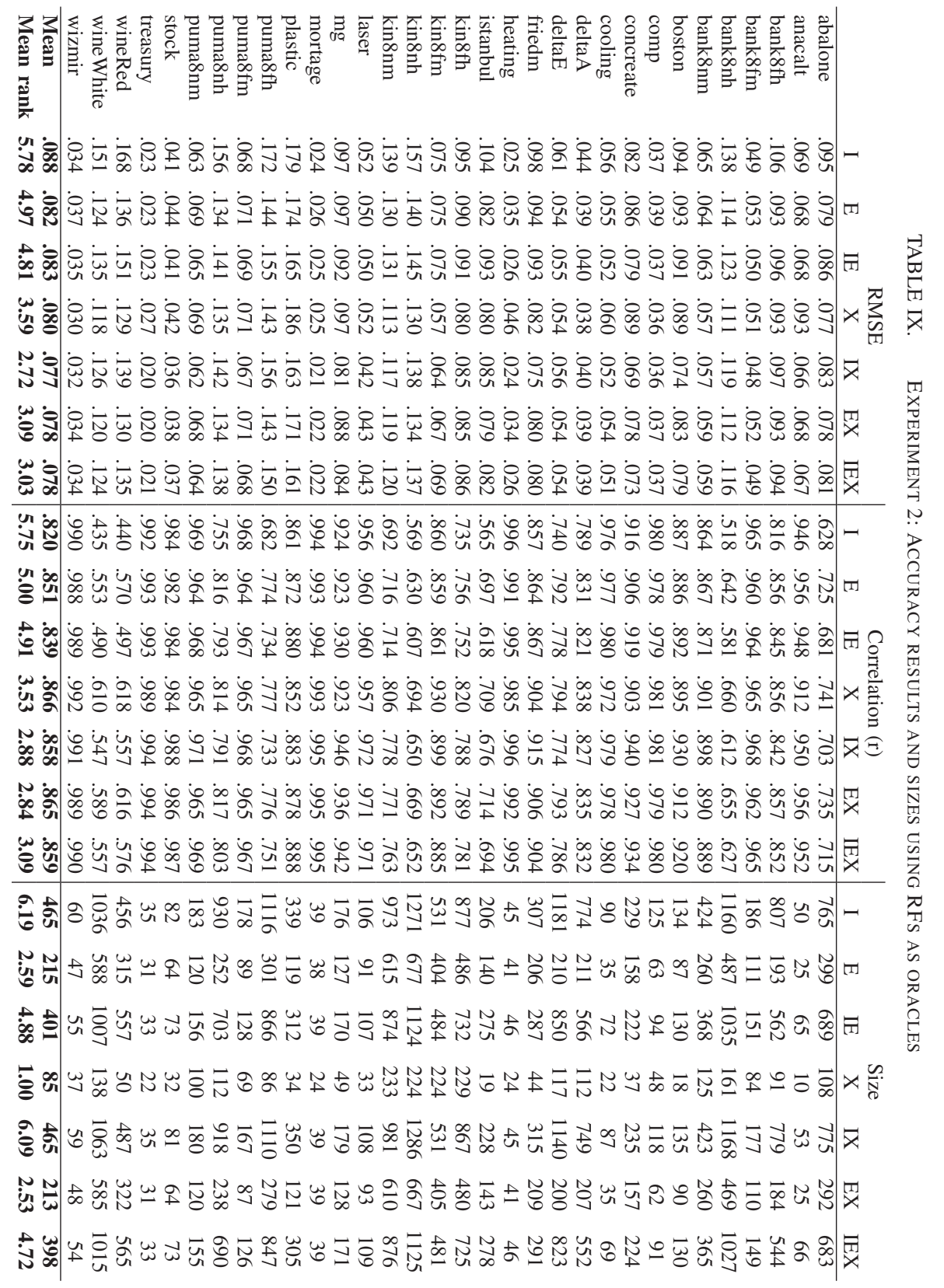

